\title{
MEJORA DE LA VELOCIDAD DE PROCESAMIENTO EN PACIENTES CON FIBROMIALGIA: DISEÑO Y APLICACIÓN DE UN PROGRAMA DE INTERVENCIÓN COGNITIVA
}

\author{
IMPROVEMENT OF PROCESSING SPEED IN PATIENTS WITH \\ FIBROMYALGIA: DESIGN AND APPLICATION OF A COGNITIVE \\ INTERVENTION PROGRAM
}

\author{
ROBERTO \\ FERNANDES-MAGALHAES \\ Universidad Rey Juan Carlos. \\ España. \\ *Correspondencia: \\ roberto.fernandesđurjc.es
}

\section{ALBERTO CARPIO}

Universidad Rey Juan Carlos. España.

\section{CANDELA GONZÁLEZ}

Universidad Rey Juan Carlos. España.

\author{
MARÍA EUGENIA DE LAHOZ \\ Universidad Rey Juan Carlos \\ España.
}

\author{
DAVID FERRERA \\ Universidad Rey Juan Carlos. \\ España.
}

\section{PALOMA BARJOLA}

Universidad Rey Juan Carlos. España.

\section{IRENE PELÁEZ}

Universidad Rey Juan Carlos. España.

\section{FRANCISCO MERCADO}

Universidad Rey Juan Carlos.

España.
La evidencia creciente considera que el enlentecimiento cognitivo en los pacientes con fibromialgia constituye uno de sus principales problemas. Este enlentecimiento, unido a la sintomatología física y afectiva que les caracteriza, afecta de manera significativa a su calidad de vida. El objetivo principal del presente estudio fue diseñar y aplicar un programa de estimulación cognitiva para comprobar sus efectos sobre la mejora de la velocidad de procesamiento de información (VPI), y como esta mejora, influye sobre otros síntomas clínicos en pacientes con fibromialgia. 22 pacientes formaron parte del programa. El programa estuvo compuesto por 8 sesiones, que incluyeron tareas de cancelación, rastreo visual, asociación y fluidez verbal. De manera previa al inicio del programa, se realizó una evaluación neuropsicológica individualizada de la VPI a través de distintas pruebas estandarizadas. Adicionalmente, se aplicaron distintos cuestionarios clínicos autoinformados para evaluar la sintomatología física (dolor y fatiga) y psicológica lansiedad, depresión, pensamientos catastróficos e impacto de la enfermedad sobre las actividades instrumentales). Dicha evaluación se volvió a aplicar al finalizar el programa, así como cinco meses después, a modo de seguimiento. Los resultados de los ANOVAs mostraron una mejora significativa en todas las pruebas de evaluación neuropsicológica aplicadas. Además, la sintomatología depresiva y el impacto de la enfermedad sobre las actividades instrumentales de la vida diaria también se vieron mejoradas. Estos resultados se mantuvieron estables cinco meses después del programa. La aplicación del presente programa de estimulación cognitiva ha mostrado ser efectivo para la mejora de la VPI en pacientes con fibromialgia. Además, esta mejora de la VPI en las pacientes, tuvo un impacto directo sobre la sintomatología depresiva y las actividades instrumentales de la vida diaria, lo que sugiere una estrecha relación entre los síntomas cognitivos y afectivos en el curso de la enfermedad.

Palabras clave: Disfunción cognitiva. Fibromialgia. Neuropsicología. Síntomas afectivos. Velocidad de Procesamiento.
Growing evidence indicates that cognitive slowness in fibromyalgia patients constitutes one of the main concerns. This slowing, together with the physical and affective symptomatology that characterises them, significantly affects their quality of life. The main objective of the present study was to design and apply a cognitive training program to test its effects on the improvement of speed processing information (SPI) in fibromyalgia, and how this improvement influences other clinical symptoms in patients with fibromyalgia. 22 patients took part of this rehabilitation program. It consisted of 8 sessions including several types of tasks: cancellation, visual search, association and verbal fluency tasks. Before starting the training program, an individualized neuropsychological assessment of the SPI was performed through standardized tests. Additionally, different self-reported clinical questionnaires were applied to assess physical (pain and fatigue) and affective (anxiety, depression, catastrophic thoughts and impact of the disease on instrumental activities) symptomatology. This evaluation was administered again once the program was completed, as well as five months later, as a follow-up. ANOVAs showed a significant improvement in all neuropsychological assessment tests. In addition, both the depressive states and the impact of the disease on the instrumental activities were improved. These effects remained stable after five months. The application of this neuropsychological rehabilitation program has shown to be effective for improving SPI processes in patients with fibromyalgia. Moreover, this improvement of IPV had a direct impact on depressive symptomatology and instrumental activities of daily living, suggesting a strong relationship between cognitive and affective symptoms in the course of the disease.

Keywords: Cognitive dysfunction; Fibromyalgia; Neuropsychology; Affective symptoms; Processing speed. 


\section{INTRODUCCIÓN}

La fibromialgia constituye un síndrome de sensibilización central caracterizado por la presencia de dolor crónico generalizado y difuso (Coppieters et al., 2015; Napadow \& Harris, 2014; Wolfe et al., 2016). La prevalencia de la enfermedad se ha estimado entre un 2-4\% de la población mundial (Wolfe et al., 2013), con una incidencia mayor en mujeres (en torno al $80 \%$ ) (Katz et al., 2010; Wolfe et al., 1995, 2018). En España, se ha estimado una prevalencia en torno al 2.3-2.4\% (Branco et al., 2010; Carmona, 2001; Collado et al., 2014; Font Gayà et al., 2020), con especial relevancia en mujeres de mediana edad, asentadas en entornos o zonas rurales (Font Gayà et al., 2020; Mas et al., 2008), y niveles educativos y socioeconómicos bajos (Mas et al., 2008; Topbas et al., 2005; White et al., 1999). Además del dolor, las pacientes suelen presentar una gran sintomatología de carácter variable (Okifuji \& Turk, 2002; Wolfe et al., 2010). Principalmente, se han observado comorbilidades afectivas como elevados niveles de ansiedad y/o depresión (Fietta et al., 2007; Galvez-Sánchez et al., 2018), así como déficits cognitivos en distintos dominios, tales como la atención, la memoria y las funciones ejecutivas (Bell et al., 2018; Ferrera et al., 2020; Wu et al., 2018). Este espectro tan amplio de sintomatología, produce una sensación de ralentización mental o problemas en la velocidad de procesamiento de la información (Arnold et al., 2008), que, junto a las quejas cognitivas (PidalMiranda et al., 2018), denominadas "fibrofog" (Glass, 2010; Kravitz \& Katz, 2015), afectan de manera significativa a la calidad de vida de los pacientes (Arnold et al., 2008; Coppieters et al., 2015; Kratz et al., 2020).

A pesar de la ausencia de consenso en su conceptualización (DeLuca, 2008; Posthuma \& de Geus, 2008; Rios-Lago
\& Periañez, 2010), la velocidad de procesamiento de la información (VPI), ha sido definida como el tiempo necesario en percibir y procesar una información, con el objetivo de preparar y ejecutar una respuesta (RiosLago \& Periañez, 2010). Se trata de un dominio cognitivo de gran relevancia, ya que por su función y localización cerebral, ampliamente distribuida, subyace y contribuye al funcionamiento cognitivo global (Posthuma \& de Geus, 2008; Ríos et al., 2004). Anatómicamente, la VPI se ha relacionado principalmente con la sustancia blanca (Debette \& Markus, 2010; Freeze et al., 2020; Kuznetsova et al., 2016), siendo el diámetro axonal, la integridad de las vainas de mielina y la eficiencia de la neurotransmisión sináptica, aspectos estructurales y funcionales básicos de este mecanismo (Rios-Lago \& Periañez, 2010). Mientras algunos estudios han descrito la VPI como un sistema de procesamiento específico, que subyace y se asocia, independientemente, con cada uno de los procesos cognitivos de orden superior (Ackerman et al., 2002; Babcock et al., 1997; Harvey, 2019), otros lo han considerado como un sistema de procesamiento global, común a todas las operaciones mentales, constituyendo un recurso general del sistema (Kail \& Salthouse, 1994; Salthouse \& Madden, 2008). Por otro lado, se podría considerar la existencia de una VPI simple, donde el procesamiento de la información requiere que se perciba y reconozca el estímulo para ejecutar una respuesta motora simple, y una VPI compleja, donde, además de ejecutar una respuesta, se requiera manipular la información (Chiaravalloti et al., 2003). En consecuencia, la VPI estaría formada por un componente sensoriomotor y un componente cognitivo o central, difícilmente disociables de procesos de orden superior, como la memoria operativa o el control 
atencional (Harvey, 2019; Myerson et al., 2003). Los modelos neuropsicológicos actuales consideran la VPI como una función del sistema (Tirapu-Ustárroz, Javier \& Herreras, 2018), estrechamente relacionada con la atención y las funciones ejecutivas (Javier TirapuUstárroz et al., 2017, 2018).

A pesar de que diversas investigaciones ponen de manifiesto la existencia de un enlentecimiento global (Alanoğlu et al., 2005; C-oté \& Moldofsky, 1997; Lee et al., 2010; Miró et al., 2011; Munguía-izquierdo et al., 2008) y específico (Leavitt \& Katz, 2008, 2012; Reyes del Paso et al., 2012; Veldhuijzen et al., 2012), en el desarrollo de distintas tareas cognitivas por parte de los pacientes con fibromialgia (Fernandes-Magalhaes et al., 2022), los resultados obtenidos sobre su estudio son mixtos (D. C. Park et al., 2001). La evidencia más consistente relacionada con la disminución de la VPI en estas pacientes, muestra que principalmente, este enlentecimiento se asocia a componentes centrales de orden superior, como la memoria de trabajo (Seo et al., 2012), los procesos de inhibición (Veldhuijzen et al., 2012), la fluidez verbal (Leavitt \& Katz, 2008, 2012) o el tiempo de planificación (Reyes del Paso et al., 2012). Además, a pesar de la existencia de un rendimiento conductual eficaz en este tipo de tareas, equiparado en algunas de ellas a participantes sin patología (Mercado et al., 2021; Peláez et al., 2019), se han observado mayores patrones de activación neural en los pacientes con fibromialgia (Fernandes-Magalhaes et al., 2022; Mercado et al., 2013), que han sido relacionados con una menor eficiencia y un mayor tiempo de respuesta a la hora de realizar distintas actividades (Mercado, Barjola, et al., 2013; Neubauer et al., 2002). Recientemente, algunos autores han relacionado estos déficits cognitivos, con la sintomatología afectiva característica de las pacientes (Galvez-Sánchez et al., 2018; Mercado et al., 2021). En este sentido, algunos programas de entrenamiento cognitivo en fibromialgia han sugerido que la mejora de distintos dominios cognitivos puede atenuar ciertos síntomas clínicos (Baker et al., 2018).

Las dificultades para disociar la VPI de otros procesos cognitivos de orden superior, obstaculizan en gran medida el proceso de evaluación. El abordaje del mismo debe realizarse a través de pruebas estandarizadas, donde el tiempo de reacción sea una variable cuantitativamente medida (Rios-Lago \& Periañez, 2010). Para ello, pruebas como Clave de Números y Búsqueda de Símbolos de la batería WAIS-IV (Wechsler, 2008), el Trail Maiking Test (Reitan, 1958), las condiciones palabra y color del Test de Stroop (Stroop, 1992), o el Finger Tapping Test (Ream, 1922), se convierten en instrumentos objetivos de gran utilidad (Ríos et al., 2004; J Tirapu-Ustárroz et al., 2008).

Aunque las alteraciones ligadas al enlentecimiento cognitivo global de las pacientes provocan un gran impacto sobre su calidad de vida (Arnold et al., 2008; Kratz et al., 2020), la escasez de investigaciones específicas que pongan en relación las dificultades de VPI en fibromialgia, con los efectos de programas específicos de estimulación cognitiva, evidencian la necesidad de profundizar en su estudio. Por ello, el objetivo principal de la presente investigación fue desarrollar y aplicar un programa integral de estimulación cognitiva para la mejora de la VPI en pacientes con fibromialgia. Debido a las características del programa, se espera encontrar beneficios ligados a los distintos componentes de la VPI (simple vs compleja). Además, la estrecha relación entre la VPI y distintos estados afectivos como la depresión (Lubrini et al., 2012), se verá reflejado en una mejora del 
estado de ánimo de las pacientes, lo que repercutiría de manera beneficiosa sobre el impacto que ejerce la enfermedad en las actividades de la vida diaria.

\section{METODOLOGÍA}

\section{Sujetos}

La muestra inicial estuvo compuesta por 30 mujeres con un rango de edad comprendido entre 38-77 años, y una media de 51,52 años (DT=8,897). Todas las participantes cumplieron con los criterios diagnósticos establecidos por la American College of Rheumatology (Wolfe et al., $1990,2016)$, con un tiempo medio desde el inicio del diagnóstico de la enfermedad de 8,71 años (DT= 5,506). Las participantes fueron reclutadas a través de la Asociación de Fibromialgia y Síndrome de Fatiga Crónica de la Comunidad de Madrid (AFINSYFACRO) y de la Asociación de Fibromialgia de Afectados de Pinto (AFAP). La mayoría de las pacientes se encontraban bajo tratamiento farmacológico $(50 \%$ ansiolíticos, $54 \%$ antidepresivos y $68 \%$ analgésicos) en el momento del estudio, no siendo posible detener su consumo para la investigación, debido tanto a motivos éticos como de prescripción médica. La participación en el estudio requirió un diagnóstico primario de fibromialgia establecido, ser mujer, hispano-hablante, y mayor de edad. Además, como criterios de exclusión, se valoró la inexistencia de trastornos neurológicos y/o psiquiátricos, y unas capacidades visuales y auditivas plenas o corregidas. Finalmente, en los análisis estadísticos se incluyeron únicamente los datos de los pacientes que participaron en un número de sesiones igual o superior al $75 \%$ del total de sesiones del entrenamiento. Debido a ello, la muestra final estuvo compuesta por 22 pacientes.

\section{Instrumentos de evaluación neuropsicológica}

Los instrumentos de evaluación neuropsicológica aplicados fueron seleccionados en base a los siguientes criterios: 1) herramientas que permitieran la evaluación directa de (a VPI, 2) que incluyeran baremos en población española, con instrucciones claras y precisas, y 3) que permitieran medir las habilidades entrenadas en el programa de intervención. Para ello, se utilizaron las pruebas neuropsicológicas que se describen a continuación.

Trail Making Test (Reitan, 1958): Test neuropsicológico utilizado para evaluar de manera breve procesos de atención alternante (Bowie \& Harvey, 2006). Consta de dos subpruebas, en la primera parte $(A)$ intervienen procesos de rastreo visual (unión numérica ascendente), en la segunda parte (B) intervienen, además, procesos de atención alternante lunión ascendente alternando números y letras). Se trata de una prueba ampliamente baremada en población española (Iñesta et al., 2021; Pena-Casanova et al., 2009; Tamayo et al., 2012) mLa medición del tiempo empleado por el sujeto en la realización de la prueba la convierten en una medida sensible de VPI (MacPherson et al., 2017).

Stroop Test (Stroop, 1992): Test neuropsicológico orientado a la evaluación de las funciones ejecutivas, como la velocidad de denominación, la inhibición de respuesta, la flexibilidad cognitiva y el control atencional IWilliams et al., 1996). Está compuesto por tres condiciones. La condición (1) palabra (P), (2) color (C) y (3) palabra/color (PC), donde el objetivo es leer la palabra escrita para la condición palabra y el color para las condiciones color y palabra/color. Se encuentra ampliamente baremada en población española (Rivera \& Arango-Lasprilla, 2017; Rognoni et al., 2013). El número de elementos 
procesados y respondidos correctamente en 45 segundos para las condiciones más básicas de palabra (1) y color (2) permiten establecer una medida sensible de VPI (Denney \& Lynch, 2009).

Versión informatizada del Finger Tapping Test (Ream, 1922), diseñada por Sybu Data (Pty) LDT (Ciudad del Cabo, Sudáfrica; www.sybu.co.za): Prueba neuropsicológica que evalúa velocidad y control motor (Mitrushina et al., 2005; Shimoyama, 1990). El objetivo es presionar una tecla durante diez segundos. Se realizan 5 ensayos alternos con cada mano. El promedio de elementos respondidos permiten establecer una medida sensible de VPI simple (Fong et al., 2009; Levitt et al., 2006; Vance et al., 2012). Se encuentra baremada en población hispano-hablante (Heaton et al., 2021).

Subtest de Busqueda de símbolos y Clave de números de la Escala de Inteligencia de Wechsler para Adultos-IV (Wechsler, 2008): Escala de Inteligencia para evaluar procesos cognitivos en personas entre 16 y 89 años. Consta de 10 subpruebas que se agrupan en cuatro índices (comprensión verbal, razonamiento perceptivo, memoria de trabajo y velocidad de procesamiento). Los subtests de búsqueda de símbolos y clave de números se asocian con procesos como el rastreo visual y la coordinación visomotora (Wechsler, 2008). El número de elementos procesados y respondidos correctamente en 120 segundos y la combinación de ambas subpruebas permiten establecer un índice de VPI ICullum \& Larrabee, 2010; Wechsler, 2008).

Con el objetivo de medir ampliamente procesos de VPI, se diseñó un paradigma experimental específico para el estudio y evaluación de la VPI, que consistió en la aplicación de una tarea de detección de estímulos (E-R) desarrollada mediante el software E-prime. Las participantes se sentaban delante de un ordenador, situado a una distancia de $70 \mathrm{~cm}$ aproximadamente. Los ensayos comenzaban con una cruz de fijación en el centro de la pantalla. Con el fin de evitar sesgos de respuesta asociados al tiempo de exposición, se utilizó un tiempo de presentación con un intervalo variable 14000 $\pm 2000 \mathrm{~ms}$ ). Inmediatamente después, se presentaba el estímulo diana (pantalla en verde). Las participantes debían responder presionando la tecla "ESPACIO", de la manera más rápida posible. El estímulo diana se mantenía fijo hasta la emisión de una respuesta por parte de las pacientes. La tarea estuvo compuesta por un total de 25 ensayos. La duración total de la tarea fue de 3 minutos aproximadamente. El promedio del tiempo de reacción en este tipo de tareas permite evaluar procesos de velocidad y coordinación motora (Chiaravalloti et al., 2003).

Adicionalmente, teniendo en cuenta la naturaleza multifactorial de la enfermedad, así como las distintas comorbilidades clínicas y afectivas que ejercen un impacto sobre la cognición en fibromialgia, se utilizaron una serie de cuestionarios autoinformados para su evaluación. El dolor y fatiga percibidos, se evaluaron a través de Escalas Visuales Analógicas (EVAs). Esta herramienta cuenta con una excelente reproducibilidad entre observadores (Breivika, 2016), y permite medir la intensidad del dolor que percibe el paciente a través una línea horizontal de 10 centímetros, en cuyos extremos se encuentran las expresiones extremas de un síntoma (Hawker et al., 2011). Además, se evaluaron otros síntomas clínicos característicos de los pacientes con fibromialgia como la depresión, mediante el Inventario de Depresión de Beck (BDI) (Beck, 1961), y la ansiedad estado y rasgo mediante el Inventario de Ansiedad Estado-Rasgo (STAI) (Spielberger 
et al., 1982). Adicionalmente, las pacientes completaron la Escala de Catastrofismo al Dolor (ECD) (Sullivan et al., 1995) y el Cuestionario de Impacto de la Fibromialgia (FIQ) (Burckhardt et al., 1991), para valorar nivel de salud actual asociado a actividades instrumentales en fibromialgia.

\section{Procedimiento}

El estudio fue aprobado por el Comité de Ética para la Investigación de la Universidad Rey Juan Carlos. Todas las participantes fueron informadas del procedimiento a llevar a cabo, firmando el consentimiento informado antes del inicio del programa. Cuatro semanas antes del inicio del programa de intervención se realizó la sesión inicial de evaluación neuropsicológica y clínica. Ésta se llevó a cabo en una sala acondicionada para el estudio, con unas condiciones óptimas de aislamiento acústico y de climatización. La duración de esta sesión fue de 1 hora aproximadamente. La distancia entre el evaluador y los sujetos fue de $100 \mathrm{~cm}$ aproximadamente.

Cuatro semanas después de la evaluación inicial, dio comienzo el programa de entrenamiento. Este consistió en 8 sesiones de una hora de duración, distribuidas a lo largo de tres semanas (2-3 sesiones semanales). Las tareas realizadas se agruparon en cuatro categorías principales, que iban aumentando en nivel de complejidad: 1) Rastreo visual, dónde fundamentalmente las actividades se encontraban relacionadas con la velocidad de rastreo visual y ejecución motora; 2) Cancelación, que incluyó actividades relacionadas con velocidad perceptiva y ejecución motora, mientras se inhibía información irrelevante para la tarea en curso; 3) Lenguaje, implicando tareas relacionadas con la velocidad de denominación y acceso al léxico; y 4) Asociación, a través de tareas relacionadas con velocidad de discriminación visual y acceso semántico. Con el objetivo de aumentar el nivel de dificultad de las tareas, se modificaron los tiempos de exposición, estableciendo 15-20 segundos en los niveles iniciales (1-2) y 5-10 segundos en los últimos niveles (3-4). En la tabla 1 se detallan las actividades realizadas y objetivos establecidos a lo largo de cada una de las 8 sesiones del programa de rehabilitación. Antes y después de cada sesión registraron los niveles de dolor y fatiga, a través de la aplicación de las EVAs.

Tras la finalización del programa, se volvieron aplicar las mismas pruebas de evaluación neuropsicológica y clínica que las pacientes completaron durante la evaluación inicial (véase apartado de instrumentos de evaluación). Asimismo, se realizó una evaluación de seguimiento 5 meses después de la finalización del programa, aplicando de nuevo las pruebas descritas anteriormente. 
Tabla 1. Actividades realizadas y objetivos establecidos para cada una de las sesiones de entrenamiento. Entre paréntesis se muestra el nivel de dificultad de la tarea.

\begin{tabular}{|c|c|c|}
\hline SESIÓN & TAREAS REALIZADAS & OBJETIVOS DE LA SESIÓN \\
\hline 1 & $\begin{array}{l}\text { Diferencias }(1-2)^{*} \\
\text { Matrices }(1-2)^{*} \\
\text { Formación de palabras }(1-2)^{*} \\
\text { Completar frases }(1-2)^{*}\end{array}$ & $\begin{array}{l}\text { V. de rastreo y discriminación visual } \\
\text { V. de denominación } \\
\text { V. de acceso al léxico }\end{array}$ \\
\hline 2 & $\begin{array}{l}\text { Asociación de iguales }(1-2)^{*} \\
\text { Asociación sílaba-imagen }(1-2)^{*} \\
\text { Cancelación de formas }(1-2)^{*}\end{array}$ & $\begin{array}{l}\text { V. de rastreo y discriminación visual } \\
\text { V. en la memoria de trabajo }\end{array}$ \\
\hline 3 & $\begin{array}{l}\text { Diferencias }(3-4)^{* *} \\
\text { Matrices }(3-4) * * \\
\text { Pseudopalabras }(1-2) * \\
\text { Completar frases }(3-4) * * \\
\text { Matriz palabra-imagen }(1-2) *\end{array}$ & $\begin{array}{l}\text { V. de rastreo y discriminación visual } \\
\text { V. en la memoria de trabajo } \\
\text { V. de acceso al léxico } \\
\text { V. de denominación }\end{array}$ \\
\hline 4 & $\begin{array}{l}\text { Asociación de iguales (3-4)** } \\
\text { Asociación sílaba-imagen (3-4)** } \\
\text { Asociación imagen-sílaba (1-2)* } \\
\text { Cancelación letra-palabra (1-2) * }\end{array}$ & $\begin{array}{l}\text { V. de rastreo y discriminación visual } \\
\text { V. de denominación } \\
\text { V. de memoria de trabajo }\end{array}$ \\
\hline 5 & $\begin{array}{l}\text { Formación de palabras }(3-4)^{* *} \\
\text { Tarea de pseudopalabras }(3-4)^{* *} \\
\text { Matriz palabra-imagen }(3-4)^{* *} \\
\text { Cancelación símbolos }(1-2)^{*}\end{array}$ & $\begin{array}{l}\text { V. de acceso al léxico } \\
\text { V. de rastreo y discriminación visual } \\
\text { V. de memoria de trabajo }\end{array}$ \\
\hline 6 & $\begin{array}{l}\text { Asociación imagen-sílaba (3-4) ** } \\
\text { Asociación figura-silueta }(1-2)^{*} \\
\text { Cancelación letra (3-4)** } \\
\text { Cancelación desordenada (1-2)* }\end{array}$ & $\begin{array}{l}\text { V. de rastreo y discriminación visual } \\
\text { V. de planificación }\end{array}$ \\
\hline 7 & $\begin{array}{l}\text { Asociación figura-silueta (3-4) ** } \\
\text { Comparación de imágenes (3-4) ** } \\
\text { Cancelación símbolos (3-4) ** } \\
\text { Cancelación palabra (3-4) ** } \\
\text { Cancelación desordenada (3-4) ** }\end{array}$ & $\begin{array}{l}\text { V. de rastreo y discriminación visual } \\
\text { V. de memoria de trabajo } \\
\text { V. de planificación }\end{array}$ \\
\hline 8 & $\begin{array}{l}\text { Tareas de rastreo visual } \\
\text { Tareas de rastreo auditivo }\end{array}$ & $\begin{array}{l}\text { V. de rastreo y discriminación visual y } \\
\text { auditivo }\end{array}$ \\
\hline
\end{tabular}

*(1-2): 20-15 segundos de respuesta. ** (3-4): 10-5 segundos de respuesta 


\section{Análisis estadístico}

La distribución de las variables dependientes fue testada usando la prueba de Kolgomorov-Smirnov. Este índice no violó la asunción de normalidad (Ryu, 2011).

Inicialmente, se compararon los resultados de la evaluación neuropsicológica aplicada antes del inicio del programa de intervención, con el grupo de referencia y corregidos por edad y escolaridad, para aquellas pruebas con baremos específicos (Búsqueda de Símbolos, Clave de Números, Índice de Velocidad de Procesamiento, Trail Making Test y Test de Stroop).

Por otro lado, con el objetivo de determinar posibles efectos del programa de entrenamiento sobre las variables neuropsicológicas (Búsqueda de Símbolos, Clave de Números, Índice de Velocidad de Procesamiento, Trail Making Test, Test de Stroop, Finger Tapping Test y Tarea de Detección de Estímulos), se realizaron ANOVAs de medidas repetidas, donde se incluyó la variable Momento Temporal de Evaluación (tres niveles: T1, previo al programa; T2, posterior; T3, seguimiento), para medir sus efectos sobre el rendimiento en las pruebas (tiempo de reacción, número de elementos procesados, puntuaciones típicas, puntuaciones escalares o índices). Además, se realizaron ANOVAs de medidas repetidas para determinar posibles efectos del programa de entrenamiento sobre las variables psicológicas (STAI, BDI, ECD) y clínicas (EVAs). En todos los contrastes, se empleó la corrección Greenhouse-Geisser (GG) épsilon para ajustar los grados de libertad del estadístico F. Las comparaciones post-hoc se realizaron para determinar entre qué condiciones se producían las diferencias, usando el procedimiento de Bonferroni (alpha <0.05). Todos los análisis se realizaron mediante el programa estadístico SPSS v. 21.0.

\section{RESULTADOS}

\section{Rendimiento inicial de las medidas neuropsicológicas de VPI}

Los resultados del rendimiento inicial en los test de evaluación neuropsicológica baremados reflejaron que las puntuaciones escalares medias para las pruebas de Búsqueda de Símbolos y Clave de Números, así como la puntuación del Índice de Velocidad de Procesamiento, se encontraron dentro de los rangos normativos del grupo de referencia. De igual manera, las puntuaciones típicas medias de las pruebas Trail Making Test-A, Test de Strooppalabra, Stroop-color, y Stroop-palabra/color, mostraron un rendimiento ajustado a los rangos normativos medios. Cabe destacar, sin embargo, que las puntuaciones típicas medias asociadas al Trail Maiking Test-B, arrojaron valores por debajo de los rangos normativos del grupo de referencia. Las medias de los tiempos de reacción y el número de aciertos en cada una de las pruebas aplicadas durante las distintas sesiones de evaluación neuropsicológica pueden observarse en la Tabla 2. 
Tabla 2. Medias y desviaciones típicas de las distintas sesiones para las pruebas de evaluación neuropsicológica aplicadas. Se informa sobre los valores de las distintas comparaciones post-hoc.

\begin{tabular}{|c|c|c|c|c|}
\hline PRUEBA & PRE & POST & SEGUIMIENTO & POST-HOC \\
\hline TMT-A (s) & $47,35(3,21)$ & $31,59(1,72)$ & $34,36(2,10)$ & $\begin{array}{l}\text { T1 vs T2: } 0,001^{* *} \\
\text { T1 vs T3: } 0,001^{* *} \\
\text { T2 vs T3: NS }\end{array}$ \\
\hline TMT-B (s) & $101,27(8,22)$ & $66,36(3,47)$ & $77,00(5,67)$ & $\begin{array}{l}\text { T1 vs T2: } 0,001^{* *} \\
\text { T1 vs T3: } 0,030^{*} \\
\text { T2 vs T3: NS }\end{array}$ \\
\hline $\mathrm{E}-\mathrm{R}$ (ms) & $345,98(18,14)$ & $298,26(10,43)$ & $319,50(10,41)$ & $\begin{array}{l}\text { T1 vs T2: } 0,042^{*} \\
\text { T1 vs T3: NS } \\
\text { T2 vs T3: } 0,061\end{array}$ \\
\hline BS (ac) & $24,95(5,32)$ & $31,63(7,57)$ & $29,68(8,49)$ & $\begin{array}{l}\text { T1 vs T2: } 0,001^{* *} \\
\text { T1 vs T3: } 0,006^{* *} \\
\text { T2 vs T3: NS }\end{array}$ \\
\hline $\mathrm{CN}$ (ac) & $55,63(12,31)$ & $66,68(12,37)$ & $63,68(13,38)$ & $\begin{array}{l}\text { T1 vs T2: } 0,001^{* *} \\
\text { T1 vs T3: } 0,009^{* *} \\
\text { T2 vs T3: NS }\end{array}$ \\
\hline Stroop-palabra (ac) & $90,45(14,61)$ & $100,41(14,74)$ & $96,68(16,15)$ & $\begin{array}{l}\text { T1 vs T2: } 0,001^{* *} \\
\text { T1 vs T3: } 0,024^{*} \\
\text { T2 vs T3: NS }\end{array}$ \\
\hline Stroop-color (ac) & $63,18(9,53)$ & $69,14(9,51)$ & $67,45(10,58)$ & $\begin{array}{l}\text { T1 vs T2: } 0,001^{* *} \\
\text { T1 vs T3: } 0,012^{*} \\
\text { T2 vs T3: NS }\end{array}$ \\
\hline
\end{tabular}




\begin{tabular}{|l|l|l|l|l|}
\hline PRUEBA & PRE & POST & SEGUIMIENTO & POST-HOC \\
\hline $\begin{array}{l}\text { Stroop-palabra/color } \\
\text { (ac) }\end{array}$ & $38,00(8,78)$ & $38,86(7,76)$ & $38,77(9,39)$ & NS \\
\hline FTT-D lac) & $58,04(8,28)$ & $61,77(6,22)$ & $60,77(7,67)$ & T1 vs T2: $0,013^{*}$ \\
\hline FTT-ND lac) & $51,07(9,57)$ & $52,20(12,96)$ & $54,86(7,93)$ & $\begin{array}{l}\text { T1 vs T3: } 0,032^{*} \\
\text { IVP (líndice) }\end{array}$ \\
\hline $95,18(14,04)$ & $106,77(14,62)$ & $103,77(15,59)$ & NS \\
\hline
\end{tabular}

T1: evaluación inicial; T2: evaluación post-intervención; T3: Evaluación de seguimiento; s: segundos; ms: milisegundos; ac: aciertos; TMT: Trail Making Test (A: Parte A; B: Parte B); E-R: Tarea de detección de estímulos; BS: Búsqueda de Símbolos; CN: Clave de Números; FTT: Finger Tapping Test (D: Mano dominante; ND: Mano no dominante); IVP: Índice de Velocidad de Procesamiento; NS: no significativo. ${ }^{*} P<.05 ;{ }^{*} P<.001$.

\section{Efectos de la intervención sobre las medidas neuropsi- cológicas de VPI}

Los ANOVAs mostraron un efecto principal del entrenamiento o estimulación cognitiva sobre el rendimiento en los subtests de Búsqueda de Símbolos $\left[F_{(1,21)}=\right.$ $\left.13,734, p=0,001 ; n_{p}^{2}=0,395\right]$ y Clave de Números $\left[F_{(1,21)}=\right.$ $\left.15,678, p=0,001 ; n_{p}^{2}=0,427\right]$. La cantidad de información correctamente procesada y ejecutada en ambas se vio incrementada en la sesión T2 $(p=0,001 ; p=0,001)$ y T3 ( $p=0,006 ; p=0,009)$, con respecto a la sesión inicial (T1). Además, el Índice de Velocidad de Procesamiento reflejó el mismo efecto $\left[F_{(1,21)}=18,107 ; p=0,001 ; n^{2}=\right.$
0,463], produciéndose una mejora durante las sesiones de evaluación T2 ( $p=0,001)$ y T3 ( $p=0,002)$, con respecto a la sesión inicial (fig.1C).

Del mismo modo, se encontró un efecto de la intervención sobre el rendimiento en las condiciones Stroop-palabra $\left[F_{(1,21)}=14,946 ; p=0,001 ; n^{2}=0,416\right]$ y Stroop-color $\left[F_{(1,21)}=10,758 ; p=0,001 ; n_{p}^{2}=0,339\right]$. La cantidad de información correctamente denominada, se vio incrementada en las sesiones T2 ( $p=0,001 ; p=0,001)$ y T3 ( $p=0,024 ; p=0,012)$ con respecto a la sesión inicial. Los resultados de los ANOVAs para la condición Strooppalabra/color, sin embargo, no arrojaron diferencias estadísticamente significativas $\left[F_{(1,21)}=0,344 ; p=0,653\right.$; $n_{p}^{2}=0,016$ ] (fig.1D).

Con respecto a la prueba Trail Making Test, los resultados mostraron un efecto de la estimulación cognitiva sobre el rendimiento en las sub-pruebas Trail Making Test- $A\left[F_{(1,21)}=28,739 ; p=0,001 ; n^{2}=0,578\right]$ y Trail Making Test- $B\left[F_{(1,21)}=23,024 ; p=0,001 ; n_{p}^{2}=0,37\right]$. Los tiempos de respuesta en la sesión T2 ( $p=0,001 ; p=0,001)$ y en la sesión T3 $(p=0,001 ; p=0,030)$ disminuyeron, con 
respecto a la sesión inicial (fig.1A).

Para el Finger Tapping Test, en la condición mano dominante (FTT-D), se encontró un incremento del rendimiento en la prueba $\left[F_{(1,21)}=6,695 ; p=0,004 ; n_{p}^{2}=\right.$ 0,242]. La cantidad de información ejecutada correctamente, se vio incrementada en la sesión de evaluación T2 $(p=0,013)$ y en la sesión T3 $(p=0,032)$ con respecto a la sesión inicial. Los resultados para la condición mano no dominante (FTT-ND), no arrojaron diferencias estadísticamente significativas $\left[F_{(1,21)}=1,60 ; p=0,219 ; n^{2}=\right.$ 0,071] (fig.1E).

Por último, los ANOVAs realizados para analizar los tiempos de reacción obtenidos en la tarea de Detección de Estímulos (E-R), también mostraron una mejora estadísticamente significativa $\left[F_{(1,21)}=4,767 ; p=0,030\right.$; $\left.\mathrm{n}_{\mathrm{p}}^{2}=0,185\right]$. Así, los tiempos de respuesta en la sesión T2 ( $p=0,042$ ), con respecto a la sesión inicial $T 1$ se vieron reducidos. Sin embargo, en la sesión T3 se atenuaron las diferencias con respecto a la sesión inicial $(p=0,413)$ (fig.1B).

\section{Efectos sobre las medidas clínicas}

Las puntuaciones medias de los cuestionarios clínicos aplicados en cada una de las sesiones de evaluación pueden observarse en la tabla 3. Los ANOVAs sobre las medidas de depresión (BDI), mostraron un efecto principal del programa de estimulación cognitiva sobre las puntuaciones totales $\left[F_{(1,21)}=6,123, p=0,011 ; n^{2}=0,226\right]$. Se produjo una reducción significativa en la sesión T2 $(p=0,009)$ y en la sesión T3 $(p=0,050)$ con respecto a la sesión inicial. Sin embargo, los ANOVAs para las puntuaciones de ansiedad (STAI), no mostraron diferencias estadísticamente significativas ni para la ansiedad estado $\left[F_{(1,21)}=2,34 ; p=0,108 ; n_{p}^{2}=0,101\right]$, ni para la
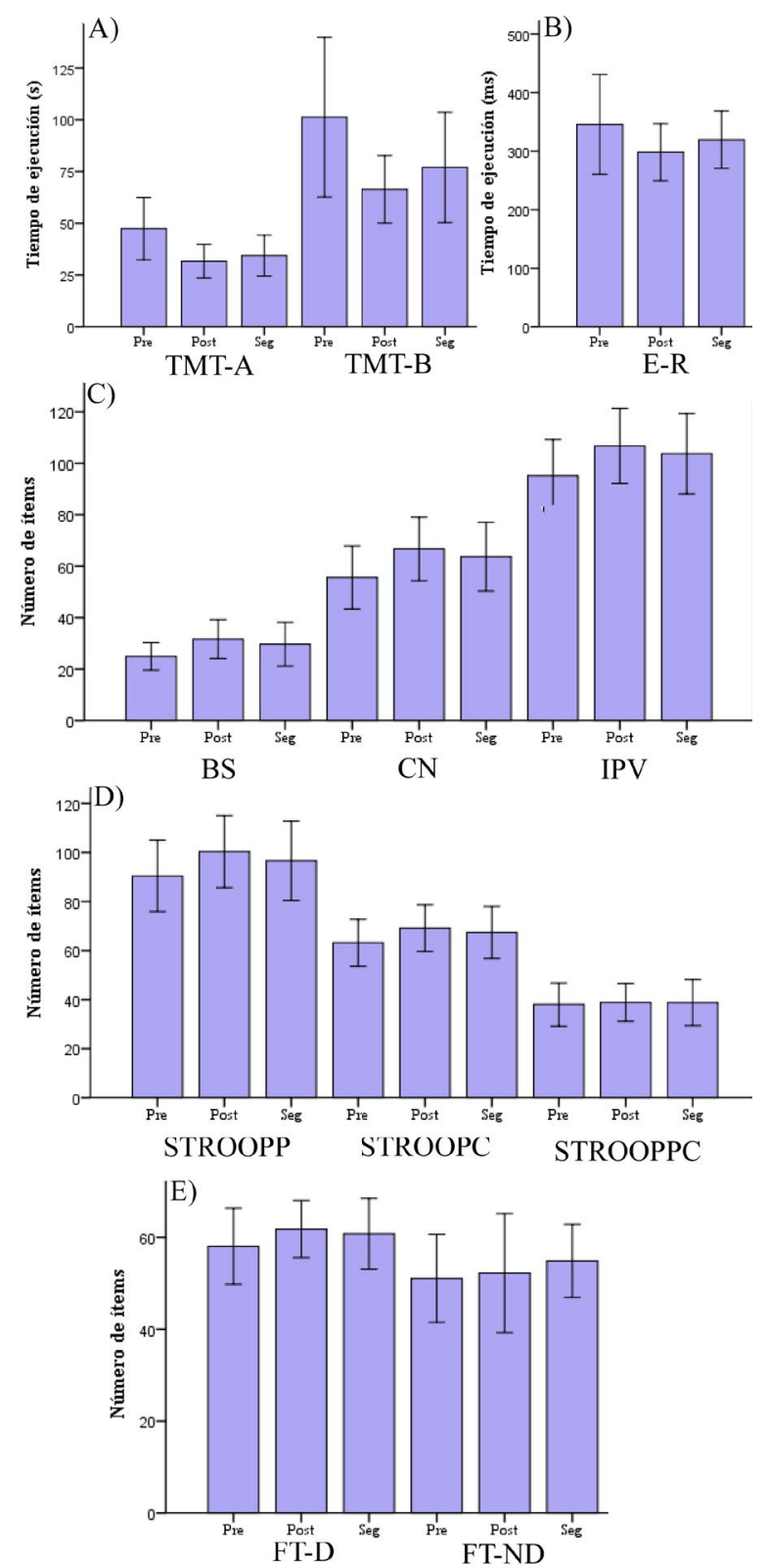

Fig. 1 Gráficas de las pruebas neuropsicológicas administradas para las sesiones pre, post y seguimiento. A) Trail Making Test A y B; B) Estimulo-Respuesta; C) Clave de Números (CN), Búsqueda de Símbolos (BS) e Índice de Velocidad de Procesamiento (IVP); D) Stroop Palabra (STROOP-P), Color (STROOP-C) y Palabra-Color (STROOP$P C)$; E) Finger Tapping Test mano dominante (FT-D) y mano no dominante (FT-ND). Las barras representan una desviación típica de la media. 
ansiedad rasgo $\left[F_{(1,21)}=2,52 ; p=0,097 ; n_{p}^{2}=0,107\right]$, a lo largo de las sesiones.

Para las puntuaciones en el cuestionario de catastrofismo (ECD), se encontró un efecto principal de la intervención $\left[F_{(1,21)}=4,72 ; p=0,017 ; n_{p}^{2}=0,184\right]$, donde solo se produjo una reducción significativa en la sesión T3 con respecto a la sesión inicial $(p=0,005)$. Concretamente, las diferencias se produjeron, para las subescalas de magnificación $\left[F_{(1,21)}=5,72 ; p=0,009 ; n_{p}^{2}=0,214\right]$ y desesperanza $\left[F_{(1,21)}=4,65 ; p=0,017 ; n_{p}^{2}=0,182\right]$. Sin embargo, la subescala de rumiación no mostró diferencias estadísticamente significativas $\left[F_{(1,21)}=2,69 ; p=0,080 ; n^{2}=0,114\right]$.

Además, se observó un efecto principal de la intervención sobre las puntuaciones en el cuestionario FIQ $\left[F_{(1,21)}=30,850 ; p=0,001 ; n_{p}^{2}=0,595\right]$, donde se produjo una reducción significativa en la sesión de evaluación T2 ( $p=0,001)$ y en la sesión de evaluación T3 ( $p=0,001)$, con respecto a la sesión inicial. Asimismo, se observó una disminución significativa en la sesión T3 con respecto a la sesión T2 ( $p=0,030)$.

Por último, los análisis estadísticos realizados sobre las escalas de fatiga y dolor (EVAs), no arrojaron diferencias significativas a lo largo de las distintas evaluaciones, ni para la variable dolor $\left[F_{(1,21)}=1,144 ; p=0,316 ; n^{2}=\right.$ $0,052]$, ni para la variable fatiga $\left[F_{(1,21)}=1,322 ; p=0,276\right.$; $\left.n_{p}^{2}=0,059\right]$. Igualmente, no se observaron diferencias estadísticamente significativas para el dolor $\left[F_{(1,21)}=\right.$ 1,$\left.777 ; p=0,197 ; n_{p}^{2}=0,078\right]$, ni para la fatiga $\left[F_{(1,21)}=\right.$ 0,$\left.198 ; p=0,661 ; n_{p}^{2}=0,009\right]$, entre las medidas tomadas al inicio y final de cada sesión.

Tabla 3. Medias y desviaciones típicas de las distintas sesiones para las pruebas de los cuestionarios autoinformados aplicados. Se informa sobre los valores de las distintas comparaciones post-hoc.

\begin{tabular}{|l|l|l|l|l|}
\hline PRUEBA & PRE & POST & SEGUIMIENTO & POST-HOC \\
\hline BDI & $20,95(1,63)$ & $17,45(1,45)$ & $17,18(1,65)$ & $\begin{array}{l}\text { T1 vs T2: } 009 * * \\
\text { T1 vs T3: } 050 * \\
\text { T2 vs T3: NS }\end{array}$ \\
\hline STAI-E & $65,41(29,06)$ & $58,50(29,06)$ & $55,09(31,04)$ & NS \\
\hline STAI-R & $78,18(21,62)$ & $71,59(21,02)$ & $74,32(25,03)$ & NS \\
\hline ECD & $56,27(6,36)$ & $48,40(5,70)$ & $44,22(5,63)$ & T1 vs T2: NS \\
\hline ECD-R & & & & T1 vs T3:, 005 \\
\hline
\end{tabular}




\begin{tabular}{|c|c|c|c|c|}
\hline PRUEBA & PRE & POST & SEGUIMIENTO & POST-HOC \\
\hline ECD-M & $63,09(25,76)$ & $56,18(23,77)$ & $52,14(24,04)$ & $\begin{array}{l}\text { T1 vs T2: NS } \\
\text { T1 vs T3: ,003 } \\
\text { T2 vs T3: NS }\end{array}$ \\
\hline ECD-D & $62,50(27,01)$ & $55,18(26,35)$ & $49,73(25,88)$ & $\begin{array}{l}\text { T1 vs T2: NS } \\
\text { T1 vs T3: ,006 } \\
\text { T2 vs T3: NS }\end{array}$ \\
\hline$F I Q$ & $71,62(12,15)$ & $56,44(13,04)$ & $48,33(17,11)$ & $\begin{array}{l}\text { T1 vs T2: }, 001^{* *} \\
\text { T1 vs T3: ,001** } \\
\text { T2 vs T3: ,030* }\end{array}$ \\
\hline EVA-D & $6,31(2,07)$ & $5,53(2,15)$ & $5,86(2,68)$ & NS \\
\hline EVA-F & $6,08(2,52)$ & $5,51(2,32)$ & $5,40(2,75)$ & NS \\
\hline EVA-DS & $5,86(2,11)$ & $5,53(2,15)$ & - & NS \\
\hline EVA-FS & $5,65(2,19)$ & $5,51(2,32)$ & - & NS \\
\hline
\end{tabular}

T1: evaluación inicial; T2: evaluación postintervención; T3: principales problemas, lo que afecta de forma signifiEvaluación de seguimiento.; BDI: Inventario de Depresión cativa a la calidad de vida de las pacientes. La ausencia de Beck; STAI: Inventario de Ansiedad Rasgo-Estado (R: de programas específicos para su mejora, ha motivado Rasgo; E: Estado); ECD: Escala de Catastrofismo al Dolor la realización del presente estudio, cuyo el objetivo (R: Rumiación; M: Magnificación; D: Desesperanza); FIQ: principal fue precisamente desarrollar y aplicar un Cuestionario de Impacto de la Fibromialgia; EVA: Escala programa de estimulación cognitiva para la mejora de Visual Analógica (D: Dolor; F: Fatiga; DS: Dolor sesiones la VPI en pacientes con fibromialgia. Aunque la mayor de entrenamiento; FS: Fatiga sesiones de entrenamiento); parte de las puntuaciones obtenidas de la evaluación NS: no significativo. ${ }^{*} P<.05 ;{ }^{*} P<.001$.

\section{DISCUSIÓN}

El enlentecimiento cognitivo o disminución de la velocidad de procesamiento de información (VPI) en las pacientes con fibromialgia constituye uno de sus neuropsicológica inicial de las pacientes se encontraron entorno al promedio en comparación con su grupo de referencia, los resultados iniciales del test Trail Making Test-B, sí reflejaron un déficit característico presente en estas pacientes. La aplicación del programa de estimulación reveló una mejora global de la VPI en toda la 
muestra, como se reflejó en los resultados asociados a la evaluación neuropsicológica realizada al finalizar el mismo. En concreto, los tiempos de ejecución se vieron significativamente reducidos en las pruebas Trail Making Test y Tarea de Detección. La cantidad de elementos correctamente procesados y ejecutados en las pruebas Stroop-palabra, Sroop-color, Búsqueda de Símbolos, Clave de Números, y Finger Tapping Test para la condición mano dominante (FTT-D), también se vio incrementada. Adicionalmente, los niveles de depresión (BDI), así como el impacto de la enfermedad sobre la vida diaria de las pacientes (FIQ), mejoraron tras el programa de intervención. Esta mejora en los distintos índices de VPI, así como la relacionada con la sintomatología afectiva y calidad de vida en las pacientes, se mantuvieron estables cinco meses después de la finalización del programa.

La evidencia más consistente vincula, principalmente, la alteración de la VPI en pacientes con fibromialgia con componentes centrales de orden superior, como el acceso al léxico o el tiempo de planificación (Leavitt \& Katz, 2008, 2012; Reyes del Paso et al., 2012; Veldhuijzen et al., 2012). Los resultados del presente estudio mostraron que las pacientes con fibromialgia mostraron un rendimiento significativamente disminuido en el test Trail Maiking Test-B. Así, mientras que la parte A del Trail Making Test se ha relacionado con procesos de rastreo visual y control motor, la parte B involucra, además, la puesta en marcha de procesos ejecutivos, tales como la flexibilidad cognitiva (Arbuthnott \& Frank, 2000). Estos procesos se asocian con la denominada VPI compleja, donde además de percibir y reconocer el estímulo, se requiere manipular la información para un uso eficiente del mismo. El rendimiento en pruebas más sencillas, como Búsqueda de Símbolos, Clave de Números, o el Test de Stroop, donde la carga cognitiva estaría relacionada, principalmente, con procesos de VPI simple, se equiparó al grupo de referencia. En esta línea, algunos autores han reflejado que las alteraciones de VPI en pacientes con fibromialgia se encuentran determinadas por la carga cognitiva de la tarea (Dick et al., 2002). Así, ante tareas complejas, como ocurre en el test Paced Auditory Serial Addition Task (PASAT), la velocidad de ejecución de los pacientes se encontró alterada, independientemente de los niveles de dolor referidos por las pacientes (Munguía-izquierdo et al., 2008). Además, a través del tiempo de ejecución en medidas que subyacen a componentes ejecutivos como la flexibilidad cognitiva o la planificación (Figura de Rey, TMT y Test del Zoo), otros autores han encontrado un rendimiento deficitario en la velocidad de ejecución de las pacientes con respecto a participantes sanos (Galvez-Sánchez et al., 2018). Estos datos apoyarían resultados previos encontrados en el estudio de otros dominios cognitivos afectados en fibromialgia como la atención, donde se ha señalado que las dificultades en el procesamiento cognitivo de los pacientes se producen, fundamentalmente, cuando la tarea es altamente demandante (Dick et al., 2002). Por tanto, la evidencia disponible apunta a que el enlentecimiento manifestado por las pacientes con fibromialgia se produciría principalmente cuando la tarea a realizar implica recursos de VPI compleja, apoyando la hipótesis que vincula dicho enlentecimiento a la realización de actividades que requieren la puesta en marcha de procesos de orden superior y alta demanda cognitiva, como ocurre con las funciones ejecutivas (Baker et al., 2018; Bell et al., 2018; Wu et al., 2018). El estudio de estas alteraciones cognitivas en fibromialgia 
resulta fundamental para el diseño e implementación de programas de rehabilitación neuropsicológica que puedan adaptarse a las necesidades específicas de los pacientes (Munguía-izquierdo et al., 2008).

Los resultados de la aplicación del programa de estimulación cognitiva mostraron una mejora de las pacientes en la mayor parte de las medidas de VPI aplicadas tras la finalización del mismo, manteniéndose los resultados estables 5 meses después. La escasez de estudios previos similares realizados en fibromialgia dificulta la generalización de los resultados, así como la comparación directa de los mismos con otras investigaciones (Baker et al., 2018). Aunque algunos estudios han señalado que la mejora de este proceso es poco consistente (de Noreña et al., 2010), otras fuentes de evidencia han revelado que los beneficios de la aplicación de estos programas se mantienen hasta 18 meses después de la intervención (Roenker et al., 2003. Asimismo, la aplicación de estimulación cognitiva a pacientes con daño cerebral adquirido ha mostrado una mejora en procesos como la VPI, la atención selectiva, o la velocidad de lectura, después de varias sesiones de entrenamiento cognitivo computarizado (Sturm \& Willmes, 1991; Wilson $\&$ Robertson, 1992). Adicionalmente, debido a su localización y distribución cerebral, así como a su estrecha relación con el resto de procesos cognitivos (Posthuma \& de Geus, 2008; Ríos et al., 2004), la mejora de la VPI, podría repercutir directamente sobre procesos como la memoria de trabajo (Duval et al., 2008) o el procesamiento atencional (Sturm \& Willmes, 1991), procesos clínicamente alterados en fibromialgia (Ferrera et al., 2020; Mercado et al., 2021)the COMT gene. Además, varias investigaciones han puesto de manifiesto una relación consistente entre la VPI y el desempeño funcional en las actividades de la vida diaria (Ball et al., 2007). Para estos autores, la mejora de la VPI, tiene un impacto directo sobre actividades funcionales como la reducción del número de movimientos peligrosos en la conducción, encontrar un número de teléfono, contar correctamente dinero o encontrar y leer los ingredientes de una receta (Ball et al., 2007). No obstante, resulta necesario llevar a cabo investigaciones más precisas que permitan arrojar luz sobre el beneficio diferencial derivado de la aplicación de este tipo de programas en pacientes con fibromialgia.

Por otra parte, hallazgos previos han destacado la presencia de una relación entre la VPI y variables afectivas como la depresión (Lubrini et al., 2012). De acuerdo a esta evidencia, los síntomas depresivos podrían modular el rendimiento neuropsicológico en algunas pruebas como el Symbol Digit Modality Test (Arnett et al., 1999). En la misma línea, se ha encontrado una relación directa entre la depresión y la VPI en pacientes con fibromialgia (Lee et al., 2010)a cardinal feature of FMS, and impaired cognition in a community setting. Men In = 3369, 40-79 years. Los resultados reflejados en el presente estudio, indicaron que no solo la sintomatología depresiva, sino también los niveles de catastrofismo, exhibieron una disminución significativa en las pacientes con posterioridad a la finalización del programa de rehabilitación, similar a lo encontrado en otros estudios (Baker et al., 2018). Estos datos aportan más evidencia sobre como la mejora de la VPI podría contribuir a la mejora de cierta sintomatología afectiva, también característica de los pacientes con fibromialgia (Fietta et al., 2007; Galvez-Sánchez et al., 2018). Además, los resultados del programa indicaron una reducción sustancial del impacto de la enfermedad, arrojando 
evidencia consistente sobre la importancia de este proceso sobre la funcionalidad en las actividades de la vida diaria, así como sobre la calidad de vida general (Arnold et al., 2008; Kratz et al., 2020). Algunos autores han sugerido que el entrenamiento cognitivo puede mejorar la cognición, estimulando los sistemas neuronales responsables de la habilidad entrenada, lo que conduce a la restauración del sistema y a un mejor desempeño de las tareas que dependen de él (Park \& Ingles, 2001), mejorando el estado afectivo de los pacientes con dolor crónico (Baker et al., 2018).

Por otro lado, cabe destacar que, a pesar de no encontrar reducciones significativas en variables como el dolor o la fatiga, estos datos deben tenerse en cuenta a la hora de interpretar los resultados. Algunos autores han señalado, como distintas variables afectivas como el miedo al dolor, pueden influir sobre los niveles de fatiga percibidos, en pacientes con fibromialgia genéticamente predispuestos (Ferrera et al., 2021)psychological symptoms and pain associated with fibromyalgia. However, how these symptoms interact to moderate genetic factors in fibromyalgia has rarely been studied to date. The present research investigates whether psychological symptoms can moderate the effects of catechol-0-methyltransferase on pain and fatigue. A total of 108 women diagnosed with fibromyalgia and 77 healthy control participants took part in the study. Pain, fatigue, and psychological symptoms lanxiety, depression, pain catastrophizing, fear of pain and fear of movement. Además, la carga cognitiva del programa, así como la sintomatología característica de la enfermedad, podrían hacer plausible un aumento en los niveles de dolor y fatiga después de las sesiones de intervención (Ickmans et al., 2015; Solberg Nes et al., 2009). Sin embargo, los datos del presente estudio indicaron que la mejora cognitiva no producía un aumento en estos niveles, manteniéndose ambos, además, estables durante seis meses. Al igual que estudios previos, nuestros datos han indicado una asociación inversa entre el nivel de fatiga y el enlentecimiento psicomotor (como es el caso del Finger Tapping Test) en las pacientes con fibromialgia (C-oté \& Moldofsky, 1997; Suhr, 2003). Para estos autores, los niveles de fatiga influyen negativamente sobre la velocidad psicomotora. Por ello, la estabilidad de los niveles de dolor y fatiga, podría considerarse un resultado favorable del programa, ya que parece existir una estrecha relación entre las demandas cognitivas de las tareas y los niveles de dolor y fatiga percibidos IIckmans et al., 2015). Este tipo de intervenciones, centradas en componentes cognitivos específicos como la VPI, pueden ayudarnos a entender el papel que ejerce este proceso sobre el funcionamiento cognitivo, afectivo y motor (Ball et al., 2007).

Entre las limitaciones del estudio se incluye el hecho de no contar con un grupo control con el que comparar directamente los efectos del programa de estimulación cognitiva. Aunque los resultados sobre el rendimiento en VPI pueden compararse con el grupo de referencia en cada uno de los test aplicados, la inclusión de un grupo control, contribuiría a establecer, con mayor precisión, la efectividad del programa de intervención (Baker et al., 2018). Unido a ello, a pesar de que la mayor parte de los instrumentos utilizados cuentan con valores normativos en población hispano-hablante, actualmente no existen datos normativos específicos de los test utilizados en población con fibromialgia. Por otro lado, a pesar de que las pacientes mantuvieron el consumo de fármacos, los resultados obtenidos en estudios previos parecen indicar que dicho factor no ha supuesto 
ninguna influencia significativa sobre el procesamiento cognitivo (Luerding et al., 2008)characterized by chronic widespread pain, stiffness and sleep disturbances. In addition, patients frequently complain of memory and attention deficits. Accumulating evidence suggests that FM is associated with CNS dysfunction and with an altered brain morphology. However, few studies have specifically investigated neuropsychological issues in patients suffering from FM. We therefore sought to determine whether neuropsychological deficits found in FM patients may be correlated with changes in local brain morphology specifically in the frontal, temporal or cingulate cortices. Twenty FM patients underwent extensive testing for potential neuropsychological deficits, which demonstrated significantly reduced working memory and impaired non-verbal long-term memory llimited to free recall condition. Por último, aunque la prevalencia de la fibromialgia es significativamente mayor en la población femenina (Katz et al., 2010; Mas et al., 2008; Wolfe et al., 2018)192 Spaniards aged 20 or above, selected by cluster sampling. Subjects were invited to a structured interview carried out by trained rheumatologists to ascertain various musculoskeletal disorders. The visit included screening and examination, validated instruments for measuring function $(\mathrm{HAQ}$, la utilización en el estudio únicamente de mujeres debe considerarse como una limitación en la generalización de los resultados presentes. Un control eficiente de la medicación, así como la inclusión de muestras formadas por hombres y mujeres en estudios futuros, podría contribuir a una mejora en el diseño de los programas de intervención.

En conclusión, la existencia de una elevada sensación de ralentización mental en pacientes con fibromialgia, y las dificultades de VPI, principalmente, en tareas asociadas a procesos complejos, convierten a esta variable en un factor relevante en los procesos de intervención. La aplicación del presente programa de estimulación cognitiva ha mostrado ser efectivo para la mejora y mantenimiento de la capacidad de VPI en pacientes con fibromialgia. Además, dada su estrecha relación con otros procesos cognitivos y afectivos, la intervención sobre su mejora puede repercutir positivamente sobre la calidad de vida de los pacientes. La combinación de este tipo de programas, con intervenciones desde otras áreas, puede ayudar a mejorar la efectividad de las terapias existentes en fibromialgia (Alemi et al., 2019; Baker et al., 2018; Burns et al., 2020; Ólason et al., 2018). Por tanto, los resultados del presente estudio sugieren que los programas de estimulación cognitiva, pueden resultar beneficiosos sobre la sintomatología cognitiva y afectiva, que caracteriza a los pacientes con fibromialgia.

\section{AGRADECIMIENTOS}

Los autores agradecen a todas las pacientes su participación en el presente estudio.

\section{FINANCIAMIENTO}

El presente trabajo se llevó a cabo gracias al apoyo de las becas PSI2017-85241-R del Ministerio de Economía y Competitividad (MINECO) del Gobierno de España, S2015/HUM-3327 EMO-CM y SAPIENTIA-CM H2019/HUM-5705 de la Comunidad de Madrid.

\section{DECLARACIÓN DE CONFLICTO DE INTERÉS}

Los autores declaran que la presente investigación se realizó en ausencia de cualquier relación comercial o económica que pudiera constituir un potencial conflicto de intereses. 


\section{BIBLIOGRAFÍA}

Ackerman, P. L., Beier, M. E., \& Boyle, M. D. (2002). Individual differences in working memory within a nomological network of cognitive and perceptual speed abilities. Journal of Experimental Psychology: General, 131(4), 567-589. https://doi.org/10.1037/0096-3445.131.4.567

Alanoğlu, E., Ulaș, U. H., Özdağ, F., Odabașı, Z., Çakçı, A., \& Vural, 0. (2005). Auditory event-related brain potentials in fibromyalgia syndrome. Rheumatology International, 25(5), 345-349. https://doi.org/10.1007/s00296-004-0443-3

Alemi, S., Malihialzackerini, S., Abolmaali Alhoseini, K., \& Khabiri, M. (2019). Comparison of the Effectiveness of Mindfulness Training and Massage-Aromatherapy on Rising Psychological Health of Elderly Women with Chronic Pain. Ranian Journal of Health Education and Health Promotion, 7(4), 359-370. https://doi.org/10.29252/ijhehp.7.4.359

Arbuthnott, K., \& Frank, J. (2000). Trail Making Test, Part B as a Measure of Executive Control: Validation Using a Set-Switching Paradigm. Journal of Clinical and Experimental Neuropsychology, 22(4), 518-528. https:// doi.org/10.1076/1380-3395(200008)22:4;1-0;FT518

Arnett, P. A., Higginson, C. I., Voss, W. D., Wright, B., Bender, W. I., Wurst, J. M., \& Tippin, J. M. (1999). Depressed mood in multiple sclerosis: Relationship to capacity-demanding memory and attentional functioning. Neuropsychology, 13(3), 434-446. https://doi.org/10.1037/0894-4105.13.3.434

Arnold, L. M., Crofford, L. J., Mease, P. J., Burgess, S. M., Palmer, S. C., Abetz, L., \& Martin, S. A. (2008). Patient perspectives on the impact of fibromyalgia. Patient Education and Counseling, 73(1), 114-120. https://doi.org/10.1016/j.pec.2008.06.005

Babcock, R. L., Laguna, K. D., \& Roesch, S. C. (1997). A comparison of the factor structure of processing speed for younger and older adults: Testing the assumption of measurement equivalence across age groups. Psychology and Aging, 12(2), 268-276. https://doi.org/10.1037/0882-7974.12.2.268

Baker, K. S., Georgiou-Karistianis, N., Lampit, A., Valenzuela, M., Gibson, S. J., \& Giummarra, M. J. (2018). Computerised training improves cognitive performance in chronic pain: a participant-blinded randomised active-controlled trial with remote supervision. Pain, 159(4), 644-655. https://doi.org/10.1097/j.pain.0000000000001150

Ball, K., Edwards, J. D., \& Ross, L. A. (2007). The Impact of Speed of Processing Training on Cognitive and Everyday Functions. The Journals of Gerontology: Series B, 62(Special_Issue_1), 19-31. https://doi. org/10.1093/geronb/62.special_issue_1.19

Beck, A. T. (1961). An Inventory for Measuring Depression. Archives of General Psychiatry, 4(6), 561. https://doi. org/10.1001/archpsyc.1961.01710120031004

Bell, T., Trost, Z., Buelow, M. T., Clay, O., Younger, J., Moore, D., \& Crowe, M. (2018). Meta-analysis of cognitive performance in fibromyalgia. Journal of Clinical and Experimental Neuropsychology, 40(7), 698-714. https://doi.org/10.1080/13803395.2017.1422699

Bowie, C. R., \& Harvey, P. D. (2006). Administration and interpretation of the Trail Making Test. Nature Protocols, 1(5), 2277-2281. https://doi.org/10.1038/nprot.2006.390

Branco, J. C., Bannwarth, B., Failde, I., Abello Carbonell, J., Blotman, F., Spaeth, M., Saraiva, F., Nacci, F., Thomas, E., Caubère, J.-P., Le Lay, K., Taieb, C., \& Matucci-Cerinic, M. (2010). Prevalence of Fibromyalgia: A Survey in Five European Countries. Seminars in Arthritis and Rheumatism, 3916), 448-453. https://doi.org/10.1016/j.semarthrit.2008.12.003

Breivika, H. (2016). Fifty years on the Visual Analogue Scale (VAS) for pain-intensity is still good for acute pain. But multidimensional assessment is needed for chronic pain. Scandinavian Journal of Pain, 11(1), 150-152. https://doi.org/10.1016/j.sjpain.2016.02.004

Burckhardt, C. S., Clark, S. R., \& Bennett, R. M. (1991). The fibromyalgia impact questionnaire: development and validation. Journal of Rheumatoly, 18(5), 728-733.

Burns, J. W., Van Dyke, B. P., Newman, A. K., Morais, C. A., \& Thorn, B. E. (2020). Cognitive behavioral therapy (CBT) and pain education for people with chronic pain: Tests of treatment mechanisms. Journal of Consulting and Clinical Psychology, 88(11), 1008-1018. https://doi.org/10.1037/ccp0000612

C-oté, K. A., \& Moldofsky, H. (1997). Sleep, daytime symptoms, and cognitive performance in patients with fibromyalgia. 
The Journal of Rheumatology, 24(10), 2014-2023. http://www.ncbi.nlm.nih.gov/pubmed/9330947

Carmona, L. (2001). The burden of musculoskeletal diseases in the general population of Spain: results from a national survey. Annals of the Rheumatic Diseases, 60(11), 1040-1045. https://doi.org/10.1136/ard.60.11.1040

Chiaravalloti, N. D., Christodoulou, C., Demaree, H. A., \& DeLuca, J. (2003). Differentiating Simple Versus Complex Processing Speed: Influence on New Learning and Memory Performance. Journal of Clinical and Experimental Neuropsychology (Neuropsychology, Development and Cognition: Section A), 25(4), 489-501. https://doi.org/10.1076/jcen.25.4.489.13878

Collado, A., Gomez, E., Coscolla, R., Sunyol, R., Solé, E., Rivera, J., Altarriba, E., Carbonell, J., \& Castells, X. (2014). Work, family and social environment in patients with Fibromyalgia in Spain: an epidemiological study: EPIFFAC study. BMC Health Services Research, 14(1), 513. https://doi.org/10.1186/s12913-014-0513-5

Coppieters, I., Ickmans, K., Cagnie, B., Nijs, J., De Pauw, R., Noten, S., Meeus, M., Pauw, R. De, Noten, S., \& Meeus, M. (2015). Cognitive performance is related to central sensitization and health-related quality of life in patients with chronic whiplash-associated disorders and fibromyalgia. Pain Physician, 18(3), E389-E402. http://www.scopus.com/ inward/record.url?eid=2-s2.0-84929464176\&partnerl $\mathrm{D}=40$ \&md5=067e799138aeb5eab5b3eb40c1ac3467

Cullum, C. M., \& Larrabee, G. J. (2010). WAIS-IV Use in Neuropsychological Assessment. In WAIS-IV Clinical Use and Interpretation (pp. 167-187). Elsevier. https:// doi.org/10.1016/B978-0-12-375035-8.10006-0

de Noreña, D., Ríos-Lago, M., Bombín-González, I., SánchezCubillo, I., García-Molina, A., \& Tirapu-Ustárroz, J. (2010). [Effectiveness of neuropsychological rehabilitation in acquired brain injury (I): attention, processing speed, memory and language]. Revista de Neurologia, 51(11), 687-698. https://www.researchgate.net/profile/ Igor_Bombin2/publication/49636357_Effectiveness_ of_neuropsychological_rehabilitation_in_acquired_
brain_injury_I_Attention_processing_speed_memory_ and_language/links/0912f5003c8629b018000000.pdf

Debette, S., \& Markus, H. S. (2010). The clinical importance of white matter hyperintensities on brain magnetic resonance imaging: Systematic review and meta-analysis. BMJ (Online), 341(7767), 288. https://doi.org/10.1136/bmj.c3666

DeLuca, J. (2008). Information processing speed: How fast, how slow, and how come? In J. DeLuca \& J. $\mathrm{H}$. Kalmar (Eds.), Information processing speed in clinical populations (p. 266). Taylor \& Francis.

Denney, D. R., \& Lynch, S. G. (2009). The impact of multiple sclerosis on patients' performance on the Stroop Test: Processing speed versus interference. Journal of the International Neuropsychological Society, 15(3), 451-458. https://doi.org/10.1017/S1355617709090730

Dick, B., Eccleston, C., \& Crombez, G. (2002). Attentional functioning in fibromyalgia, rheumatoid arthritis, and musculoskeletal pain patients. Arthritis \& Rheumatism, 47(6), 639-644. https://doi.org/10.1002/art.10800

Duval, J., Coyette, F., \& Seron, X. (2008). Rehabilitation of the central executive component of working memory: A re-organisation approach applied to a single case. Neuropsychological Rehabilitation, 18(4), 430-460. https://doi.org/10.1080/09602010701573950

Fernandes-Magalhaes, R., Ferrera, D., Peláez, I., Martín-Buro, M. C., Carpio, A., De Lahoz, M. E., Barjola, P., \& Mercado, F. (2022). Neural correlates of the attentional bias towards pain-related faces in fibromyalgia patients: An ERP study using a dot-probe task. Neuropsychologia, 166, 108141. https://doi.org/10.1016/j.neuropsychologia.2021.108141

Ferrera, D., Gómez-Esquer, F., Peláez, I., Barjola, P., Fernandes-Magalhaes, R., Carpio, A., De Lahoz, M. E., Díaz-Gil, G., \& Mercado, F. (2020). Effects of COMT Genotypes on Working Memory Performance in Fibromyalgia Patients. Journal of Clinical Medicine, 9(8), 2479. https://doi.org/10.3390/jcm9082479

Ferrera, D., Mercado, F., Peláez, I., Martínez-Iñigo, D., FernandesMagalhaes, R., Barjola, P., Écija, C., Díaz-Gil, G., \& Gómez-Esquer, F. (2021). Fear of pain moderates 
the relationship between self-reported fatigue and methionine allele of catechol-0-methyltransferase gene in patients with fibromyalgia. PLOS ONE, 16(4), e0250547. https://doi.org/10.1371/journal.pone.0250547

Fietta, P., Fietta, P., \& Manganelli, P. (2007). Fibromyalgia and psychiatric disorders. Acta Biomedica de l'Ateneo Parmense, 78(2), 88-95.

Fong, K. N. K., Chan, M. K. L., Ng, P. P. K., \& Ng, S. S. W. (2009). Measuring processing speed after traumatic brain injury in the outpatient clinic1. NeuroRehabilitation, 24(2), 165-173. https://doi.org/10.3233/NRE-2009-0465

Font Gayà, T., Bordoy Ferrer, C., Juan Mas, A., Seoane-Mato, D., Álvarez Reyes, F., Delgado Sánchez, M., Martínez Dubois, C., Sánchez-Fernández, S. A., Marena Rojas Vargas, L., García Morales, P. V, Olivé, A., Rubio Muñoz, P., Larrosa, M., Navarro Ricós, N., Sánchez-Piedra, C., Díaz-González, F., Bustabad-Reyes, S., \& Working Group Proyecto EPISER2016. (2020). Prevalence of fibromyalgia and associated factors in Spain. Clinical and Experimental Rheumatology, 38 Suppl 1(1), 47-52. http://www.ncbi.nlm.nih.gov/pubmed/31928589

Freeze, W. M., Jacobs, H. I. L., de Jong, J. J., Verheggen, I. C. M., Gronenschild, E. H. B. M., Palm, W. M., Hoff, E. I., Wardlaw, J. M., Jansen, J. F. A., Verhey, F. R., \& Backes, W. H. (2020). White matter hyperintensities mediate the association between blood-brain barrier leakage and information processing speed. Neurobiology of Aging, 85, 113-122. https://doi.org/10.1016/j.neurobiolaging.2019.09.017

Galvez-Sánchez, C. M., Reyes del Paso, G. A., \& Duschek, S. (2018). Cognitive Impairments in Fibromyalgia Syndrome: Associations With Positive and Negative Affect, Alexithymia, Pain Catastrophizing and Self-Esteem. Frontiers in Psychology, 9. https://doi.org/10.3389/fpsyg.2018.00377

Glass, J. M. (2010). Cognitive dysfunction in fibromyalgia syndrome. Journal of Musculoskeletal Pain, 18(4), 367-372. https://doi.org/10.3109/10582452.2010.502626

Harvey, P. D. (2019). Domains of cognition and their assessment . Dialogues in Clinical Neuroscience, 21(3), 227-237. https://doi.org/10.31887/DCNS.2019.21.3/pharvey
Hawker, G. A., Mian, S., Kendzerska, T., \& French, M. (2011). Measures of adult pain: Visual Analog Scale for Pain IVAS Pain), Numeric Rating Scale for Pain (NRS Pain), McGill Pain Questionnaire (MPQ), Short-Form McGill Pain Questionnaire (SF-MPQ), Chronic Pain Grade Scale (CPGS), Short Form-36 Bodily Pain Scale ISF. Arthritis Care \& Research, 63(S11), S240-S252. https://doi.org/10.1002/acr.20543

Heaton, A., Gooding, A., Cherner, M., Umlauf, A., Franklin, D. R., Rivera Mindt, M., Suárez, P., Artiola i Fortuni, L., Heaton, R. K., \& Marquine, M. J. (2021). Demographicallyadjusted norms for the Grooved Pegboard and Finger Tapping tests in Spanish-speaking adults: Results from the Neuropsychological Norms for the U.S.Mexico Border Region in Spanish (NP-NUMBRS) Project. The Clinical Neuropsychologist, 35(2), 396-418. https://doi.org/10.1080/13854046.2020.1713400

Ickmans, K., Meeus, M., De Kooning, M., Lambrecht, L., Pattyn, N., \& Nijs, J. (2015). Associations between cognitive performance and pain in chronic fatigue syndrome: comorbidity with fibromyalgia does matter. Physiotherapy, 101, e635-e636. https://doi.org/10.1016/j.physio.2015.03.3465

Iñesta, C., Oltra-Cucarella, J., Bonete-López, B., Calderón-Rubio, E., \& Sitges-Maciá, E. (2021). Regression-Based Normative Data for Independent and Cognitively Active Spanish Older Adults: Digit Span, Letters and Numbers, Trail Making Test and Symbol Digit Modalities Test. International Journal of Environmental Research and Public Health, 18(19), 9958. https://doi.org/10.3390/ijerph18199958

Kail, R., \& Salthouse, T. A. (1994). Processing speed as a mental capacity. Acta Psychologica, 86(2-3), 199-225. https://doi.org/10.1016/0001-6918(94)90003-5

Katz, J. D., Mamyrova, G., Guzhva, O., \& Furmark, L. (2010). Gender bias in diagnosing fibromyalgia. Gender Medicine, 7(1), 19-27. https://doi.org/10.1016/j.genm.2010.01.003

Kratz, A. L., Whibley, D., Kim, S., Sliwinski, M., Clauw, D., \& Williams, D. A. (2020). Fibrofog in Daily Life: An Examination of Ambulatory Subjective and Objective Cognitive Function in Fibromyalgia. Arthritis Care \& Research, 72(12), 1669-1677. https://doi.org/10.1002/acr.24089 
Kravitz, H. M., \& Katz, R. S. (2015). Fibrofog and fibromyalgia: a narrative review and implications for clinical practice. Rheumatology International, 35(7), 1115-1125. https://doi.org/10.1007/s00296-014-3208-7

Kuznetsova, K. A., Maniega, S. M., Ritchie, S. J., Cox, S. R., Storkey, A. J., Starr, J. M., Wardlaw, J. M., Deary, I. J., \& Bastin, M. E. (2016). Brain white matter structure and information processing speed in healthy older age. Brain Structure and Function, 221(6), 3223-3235. https://doi.org/10.1007/s00429-015-1097-5

Leavitt, F., \& Katz, R. S. (2008). Speed of Mental Operations in Fibromyalgia. JCR: Journal of Clinical Rheumatology, PAP(4), 214-218. https://doi.org/10.1097/RHU.0b013e31817a2472

Leavitt, F., \& Katz, R. S. (2012). Lexical Memory Deficit in Fibromyalgia Syndrome. Journal of Musculoskeletal Pain, 20(2), 82-86. https://doi.org/10.3109/10582452.2012.673545

Lee, D. M., Pendleton, N., Tajar, A., O'Neill, T. W., O'Connor, D. B., Bartfai, G., Boonen, S., Casanueva, F. F., Finn, J. D., Forti, G., Giwercman, A., Han, T. S., Huhtaniemi, I. T., Kula, K., Lean, M. E. J., Punab, M., Silman, A. J., Vanderschueren, D., Moseley, C. M., ... McBeth, J. (2010). Chronic widespread pain is associated with slower cognitive processing speed in middle-aged and older European men. Pain, 151(1), 30-36. https://doi.org/10.1016/j.pain.2010.04.024

Levitt, T., Fugelsang, J., \& Crossley, M. (2006). Processing Speed, Attentional Capacity, and Age-Related Memory Change. Experimental Aging Research, 32(3), 263-295. https://doi.org/10.1080/03610730600699118

Lubrini, G., Perianez, J. A., Rios-Lago, M., \& Frank, A. (2012). Processing speed in relapsing-remitting multiple sclerosis: the role played by the depressive symptoms. Revista de Neurologia, 55(10), 585-592. http://www.ncbi.nlm.nih.gov/pubmed/23143959

Luerding, R., Weigand, T., Bogdahn, U., \& Schmidt-Wilcke, T. (2008). Working memory performance is correlated with local brain morphology in the medial frontal and anterior cingulate cortex in fibromyalgia patients: structural correlates of pain-cognition interaction. Brain, 131(12), 3222-3231. https://doi.org/10.1093/brain/awn229
MacPherson, S. E., Cox, S. R., Dickie, D. A., Karama, S., Starr, J. M., Evans, A. C., Bastin, M. E., Wardlaw, J. M., \& Deary, I. J. (2017). Processing speed and the relationship between Trail Making Test-B performance, cortical thinning and white matter microstructure in older adults. Cortex, 95, 92-103.

Mas, A. J., Carmona, L., Valverde, M., Ribas, B., \& EPISER Study Group. (2008). Prevalence and impact of fibromyalgia on function and quality of life in individuals from the general population: results from a nationwide study in Spain. Clinical and Experimental Rheumatology, 26(4), 519-526. http://www.ncbi.nlm.nih.gov/pubmed/18799079

Mercado, F., Barjola, P., Fernndez-Snchez, M., Guerra, V., \& Gmez-Esquer, F. (2013). Brain Function in Fibromyalgia: Altered Pain Processing and Cognitive Dysfunction. In Functional Brain Mapping and the Endeavor to Understand the Working Brain. InTech. https://doi.org/10.5772/56495

Mercado, F., Ferrera, D., Fernandes-Magalhaes, R., Peláez, I., \& Barjola, P. (2021). Altered Subprocesses of Working Memory in Patients with Fibromyalgia: An Event-Related Potential Study Using N -Back Task. Pain Medicine. https://doi.org/10.1093/pm/pnab190

Mercado, F., González, J. L., Barjola, P., Fernández-Sánchez, M., López-López, A., Alonso, M., \& Gómez-Esquer, F. (2013). Brain correlates of cognitive inhibition in fibromyalgia: Emotional intrusion of symptom-related words. International Journal of Psychophysiology, 88(2), 182-192. https://doi.org/10.1016/j.ijpsycho.2013.03.017

Miró, E., Lupiáñez, J., Hita, E., Martínez, M. P., Sánchez, A. I., \& Buela-Casal, G. (2011). Attentional deficits in fibromyalgia and its relationships with pain, emotional distress and sleep dysfunction complaints. Psychology and Health, 26(6), 765-780. https://doi.org/10.1080/08870446.2010.493611

Mitrushina, M., Boone, K. B., Razani, J., \& D’Elia, L. F. (2005). Handbook of normative data for neuropsychological assessment. Oxford University Press.

Munguía-izquierdo, D., Legaz-arrese, A., \& Reverter-masía, D. M. J. (2008). Munguía_izquierdo_2008.pdf. 20, 427-431.

Myerson, J., Hale, S., Zheng, Y., Jenkins, L., \& Widaman, K. F. (2003). The difference engine: A model of diversity in 
speeded cognition. Psychonomic Bulletin \& Review, 10(2), 262-288. https://doi.org/10.3758/BF03196491

Napadow, V., \& Harris, R. E. (2014). What has functional connectivity and chemical neuroimaging in fibromyalgia taught us about the mechanisms and management of 'centralized' pain? Arthritis Research \& Therapy, 16(4), 425. https://doi.org/10.1186/s13075-014-0425-0

Neubauer, A. C., Fink, A., \& Schrausser, D. G. (2002). Intelligence and neural efficiency. Intelligence, 30(6), 515-536. https://doi.org/10.1016/S0160-2896(02)00091-0

Okifuji, A., \& Turk, D. C. (2002). Stress and Psychophysiological Dysregulation in Patients With Fibromyalgia Syndrome. 27(2).

Ólason, M., Andrason, R. H., Jónsdóttir, I. H., Kristbergsdóttir, H., \& Jensen, M. P. (2018). Cognitive Behavioral Therapy for Depression and Anxiety in an Interdisciplinary Rehabilitation Program for Chronic Pain: a Randomized Controlled Trial with a 3-Year Follow-up. International Journal of Behavioral Medicine, 25(1), 55-66. https://doi.org/10.1007/s12529-017-9690-z

Park, D. C., Glass, J. M., Minear, M., \& Crofford, L. J. (2001). Cognitive Function in Fibromyalgia Patients. Arthritis \& Rheumatism, 44(9), 2125-2133. https://doi.org/10.1002/15290131(200109)44:9<2125::AID-ART365>3.0.C0;2-1

Park, N. W., \& Ingles, J. L. (2001). Effectiveness of attention rehabilitation after an acquired brain injury: $A$ meta-analysis. Neuropsychology, 15(2), 199-210. https://doi.org/10.1037/0894-4105.15.2.199

Peláez, I., Ferrera, D., Barjola, P., Fernandes, R., \& Mercado, F. (2019). Subliminal emotional pictures are capable of modulating early cerebral responses to pain in fibromyalgia. PLOS ONE, 14(6), e0217909. https:// doi.org/10.1371/journal.pone.0217909

Pena-Casanova, J., Quinones-Ubeda, S., Quintana-Aparicio, M., Aguilar, M., Badenes, D., Molinuevo, J. L., Torner, L., Robles, A., Barquero, M. S., Villanueva, C., Antunez, C., Martinez-Parra, C., Frank-Garcia, A., Sanz, A., Fernandez, M., Alfonso, V., Sol, J. M., \& Blesa, R. (2009). Spanish Multicenter Normative Studies (NEURONORMA Project): Norms for Verbal Span, Visuospatial Span, Letter and
Number Sequencing, Trail Making Test, and Symbol Digit Modalities Test. Archives of Clinical Neuropsychology, 24(4), 321-341. https://doi.org/10.1093/arclin/acp038

Pidal-Miranda, M., González-Villar, A. J., Carrillo-de-la-Peña, M. T., Andrade, E., \& Rodríguez-Salgado, D. (2018). Broad cognitive complaints but subtle objective working memory impairment in fibromyalgia patients. PeerJ, 6, e5907. https://doi.org/10.7717/peerj.5907

Posthuma, D., \& de Geus, E. (2008). The genetics of information processing speed in humans. In J. DeLuca \& J. Kalmar (Eds.), Information processing speed in clinical populations (pp. 79-101). Taylor \& Francis.

Ream, M. J. (1922). The tapping test: A measure of motility. Psychological Monographs, 31(1), 293-319. https://doi.org/10.1037/h0093175

Reitan, R. M. (1958). Validity of the Trail Making Test as an Indicator of Organic Brain Damage. Perceptual and Motor Skills, 8(3), 271-276. https://doi.org/10.2466/pms.1958.8.3.271

Reyes del Paso, G. A., Pulgar, Á., Duschek, S., \& Garrido, S. (2012). Cognitive impairment in fibromyalgia syndrome: The impact of cardiovascular regulation, pain, emotional disorders and medication. European Journal of Pain, 16(3), 421-429. https://doi.org/10.1002/j.1532-2149.2011.00032.x

Rios-Lago, M., \& Periañez, J. A. (2010). Attention and Speed of Information Processing. In G. Koob, R. F. Thompson, \& M. Le Moal (Eds.), Encyclopedia of Behavioral Neuroscience (pp. 109-117). Elsevier.

Ríos, M., Periáñez, J. A., \& Muñoz-Céspedes, J. M. (2004). Attentional control and slowness of information processing after severe traumatic brain injury. Brain Injury, 18(3), 257-272. https://doi.org/10.1080/02699050310001617442

Rivera, D., \& Arango-Lasprilla, J. C. (2017). Methodology for the development of normative data for Spanish-speaking pediatric populations. NeuroRehabilitation, 41(3), 581-592. https://doi.org/10.3233/NRE-172275

Roenker, D. L., Cissell, G. M., Ball, K. K., Wadley, V. G., \& Edwards, J. D. (2003). Speed-of-Processing and Driving Simulator Training Result in Improved Driving 
Performance. Human Factors: The Journal of the Human Factors and Ergonomics Society, 45(2), 218-233. https://doi.org/10.1518/hfes.45.2.218.27241

Rognoni, T., Casals-Coll, M., Sánchez-Benavides, G., Quintana, M., Manero, R. M., Calvo, L., Palomo, R., Aranciva, F., Tamayo, F., \& Peña-Casanova, J. (2013). Spanish normative studies in young adults (NEURONORMA young adults project): Norms for Stroop Color-Word Interference and Tower of London-Drexel University tests. Neurología (English Edition), 28(2), 73-80. https://doi.org/10.1016/j.nrleng.2012.02.004

Ryu, E. (2011). Effects of skewness and kurtosis on normaltheory based maximum likelihood test statistic in multilevel structural equation modeling. Behavior Research Methods, 43(4), 1066-1074. https://doi.org/10.3758/s13428-011-0115-7

Salthouse, T. A., \& Madden, D. J. (2008). Information Processing Speed and Aging. In J. DeLuca \& J. H. Kalmar (Eds.), Information processing speed in clinical populations (pp. 221-241). Taylor \& Francis.

Seo, J., Kim, S.-H., Kim, Y.-T., Song, H., Lee, J., Kim, S.-H., Han, S. W., Nam, E. J., Kim, S.-K., Lee, H. J., Lee, S.-J., \& Chang, Y. (2012). Working Memory Impairment in Fibromyalgia Patients Associated with Altered Frontoparietal Memory Network. PLoS ONE, 7(6), e37808. https://doi.org/10.1371/journal.pone.0037808

Shimoyama, I. (1990). The Finger-Tapping Test. Archives of Neurology, 47(6), 681. https://doi.org/10.1001/ archneur.1990.00530060095025

Solberg Nes, L., Roach, A. R., \& Segerstrom, S. C. (2009). Executive Functions, Self-Regulation, and Chronic Pain: A Review. Annals of Behavioral Medicine, 37(2), 173-183. https://doi.org/10.1007/s12160-009-9096-5

Spielberger, C. D., Gorsuch, R. L., \& Lushene, R. (1982). Manual del Cuestionario de Ansiedad Estado/Rasgo (STAl). TEA Ediciones.

Stroop, J. R. (1992). Studies of interference in serial verbal reactions. Journal of Experimental Psychology: General, 121(1), 15-23. https://doi.org/10.1037/0096-3445.121.1.15
Sturm, W., \& Willmes, K. (1991). Efficacy of a reaction training on various attentional and cognitive functions in stroke patients. Neuropsychological Rehabilitation, 1(4), 259-280. https://doi.org/10.1080/09602019108402258

Suhr, J. A. (2003). Neuropsychological impairment in fibromyalgia. Journal of Psychosomatic Research, 55(4), 321-329. https://doi.org/10.1016/S0022-3999(02)00628-1

Sullivan, M., Bishop, S., \& Pivik, J. (1995). The pain catastrophizing scale: development and validation. Psychological Assessment, 7(4), 524.

Tamayo, F., Casals-Coll, M., Sánchez-Benavides, G., Quintana, M., Manero, R. M., Rognoni, T., Calvo, L., Palomo, R., Aranciva, F., \& Peña-Casanova, J. (2012). Spanish normative studies in a young adult population (NEURONORMA young adults project): Guidelines for the span verbal, span visuo-spatial, Letter-Number Sequencing, Trail Making Test and Symbol Digit Modalities Test. Neurología (English Edition), 27(6), 319-329. https://doi.org/10.1016/j.nrleng.2012.07.008

Tirapu-Ustárroz, Javier, P. C., \& Herreras, E. B. (2018). Funciones ejecutivas en población infantil: propuesta de una clarificación conceptual e integradora basada en resultado de análisis factoriales. Cuadernos de Neuropsicología/Panamerican Journal of Neuropsychology, 12(3). https://doi.org/10.7714/CNPS/12.3.203

Tirapu-Ustárroz, J, García-Molina, A., Luna-Lario, P., RoigRovira, T., \& Pelegrín-Valero, C. (2008). [Models of executive control and functions (I)]. Revista de Neurologia, 46(11), 684-692. https://doi.org/rn2008252 [pii]

Tirapu-Ustárroz, Javier, Bausela Herreras, E., \& Cordero Andrés, P. (2018). Modelo de funciones ejecutivas basado en análisis factoriales en población infantil y escolar: Metaanálisis. Rev. Neurol, 67, 215-225.

Tirapu-Ustárroz, Javier, Cordero-Andrés, P., Luna-Lario, P., \& Hernáez-Goñi, P. (2017). Propuesta de un modelo de funciones ejecutivas basado en análisis factoriales. Revista de Neurología, 64(2), 75-84.

Topbas, M., Cakirbay, H., Gulec, H., Akgol, E., Ak, I., \& Can, G. (2005). The prevalence of fibromyalgia in women aged 20-64 
in Turkey. Scandinavian Journal of Rheumatology, 34(2), 140-144. https://doi.org/10.1080/03009740510026337

Vance, D. E., Fazeli, P. L., Ross, L. A., Wadley, V. G., \& Ball, K. K. (2012). Speed of Processing Training With Middle-Age and Older Adults With HIV: A Pilot Study. Journal of the Association of Nurses in AIDS Care, 23(6), 500-510. https://doi.org/10.1016/j.jana.2012.01.005

Veldhuijzen, D. S., Sondaal, S. F. V., \& Oosterman, J. M. (2012). Intact Cognitive Inhibition in Patients With Fibromyalgia but Evidence of Declined Processing Speed. The Journal of Pain, 13(5), 507-515. https://doi.org/10.1016/j.jpain.2012.02.011

Wechsler, D. (2008). Wechsler Adult Intelligence Scale-

Fourth Edition (WAIS-IV). Pearson.

White, K. P., Speechley, M., Harth, M., \& Ostbye, T. (1999). The London Fibromyalgia Epidemiology Study: the prevalence of fibromyalgia syndrome in London, Ontario. The Journal of Rheumatology, 26(7), 1570-1576. http://www.ncbi.nlm.nih.gov/pubmed/10405947

Williams, J. M. G., Mathews, A., \& MacLeod, C. (1996). The emotional Stroop task and psychopathology. Psychological Bulletin, 120(1), 3-24. https://doi.org/10.1037/0033-2909.120.1.3

Wilson, C., \& Robertson, I. H. (1992). A home-based intervention for attentional slips during reading following head injury: $A$ single case study. Neuropsychological Rehabilitation, 2(3), 193-205. https://doi.org/10.1080/09602019208401408

Wolfe, F., Brähler, E., Hinz, A., \& Häuser, W. (2013). Fibromyalgia Prevalence, Somatic Symptom Reporting, and the Dimensionality of Polysymptomatic Distress: Results From a Survey of the General Population. Arthritis Care \& Research, 65(5), 777-785. https://doi.org/10.1002/acr.21931

Wolfe, F., Clauw, D. J., Fitzcharles, M.-A., Goldenberg, D.

L., Häuser, W., Katz, R. L., Mease, P. J., Russell, A.

S., Russell, I. J., \& Walitt, B. (2016). 2016 Revisions to the 2010/2011 fibromyalgia diagnostic criteria. Seminars in Arthritis and Rheumatism, 46(3), 319-329. https://doi.org/10.1016/j.semarthrit.2016.08.012

Wolfe, F., Clauw, D. J., Fitzcharles, M. A., Goldenberg, D. L., Katz, R. S., Mease, P., Russell, A. S., Russell, I. J., Winfield, J. B., \&
Yunus, M. B. (2010). The American College of Rheumatology preliminary diagnostic criteria for fibromyalgia and measurement of symptom severity. Arthritis Care and Research, 62(5), 600-610. https://doi.org/10.1002/acr.20140

Wolfe, F., Ross, K., Anderson, J., Russell, I. J., \& Hebert, L. (1995). The prevalence and characteristics of fibromyalgia in the general population. Arthritis \& Rheumatism, 38(1), 19-28. https://doi.org/10.1002/art.1780380104

Wolfe, F., Smythe, H. A., Yunus, M. B., Bennett, R. M., Bombardier, C., Goldenberg, D. L., Tugwell, P., Campbell, S. M., Abeles, M., Clark, P., Fam, A. G., Farber, S. J., Fiechtner, J. J., Michael Franklin, C., Gatter, R. A., Hamaty, D., Lessard, J., Lichtbroun, A. S., Masi, A. T., ... Sheon, R. P. (1990). The american college of rheumatology 1990 criteria for the classification of fibromyalgia. Arthritis \& Rheumatism, 33(2), 160-172. https://doi.org/10.1002/art.1780330203

Wolfe, F., Walitt, B., Perrot, S., Rasker, J. J., \& Häuser, W. (2018). Fibromyalgia diagnosis and biased assessment: Sex, prevalence and bias. PLOS ONE, 13(9), e0203755. https://doi.org/10.1371/journal.pone.0203755

Wu, Y.-L., Huang, C.-J., Fang, S.-C., Ko, L.-H., \& Tsai, P.-S. (2018). Cognitive Impairment in Fibromyalgia. Psychosomatic Medicine, 80(5), 432-438. https:// doi.org/10.1097/PSY.0000000000000575 